\title{
The Research and Design of Children's Dental Treatment Machine Based on Ergonomics
}

\author{
Yingjun $\mathrm{Su}^{1}$ \\ South China University of Technology \\ Guangzhou, 510006, China \\ E-mail: suyingjun1630163. com
}

Children's dental treatment has a more complex dual man-machine interface, so this paper presents a design method base on ergonomic-prototype. It uses a virtual prototype which is easy to modify instead of the final product to do the ergonomic design and analysis, making the ergonomic theory throughout the product design process. The ergonomic-prototype is constructed base on the human body size and human working posture. It is a man-machine performance carrier, giving auxiliary reference to the designers and engineers. This practice can provide theoretical basis for the development of special children's dental treatment machine, and also verify the feasibility of the method of this system.

ISCC 2015

18-19, December, 2015

Guangzhou, China

${ }^{1}$ Speaker 


\section{Introduction}

Dental treatment machine is the important equipment for dentistry treatment, and its function, shape and other design have a direct impact on the medical treatment and the patient's health. Due to the special domestic situation, children's dental needs are increasing rapidly. Children are a special group in dental treatment. The development of child-specific treatment machine is immature with lots of deficiencies, for example, simply to reduce the size of adult one, not only does not meet the child's body proportions, but also can't meet the aesthetic standards of children. The application of ergonomics for the detailed analysis is necessary.

\section{The Research and Design Method of Treatment Machine Based on Ergonomics}

\subsection{Ergonomics}

The definition of ergonomics by the International Ergonomics Association: ergonomics is a discipline on the study of various factors of human anatomy, physiology and psychology in some work environments, on the interaction of man, machine and the environment, and on the unified consideration of work efficiency, human health, safety and comfort in daily life. The main purpose of its study is to reveal the relationship among the law of man, machine and the environment, in order to achieve human - machine - environment overall system optimization[1].

In the design of the dental treatment machine, ergonomics of the "people" mainly refers to the dentist and patient; the "machine" refers to the treatment of dental chairs and other; "environment" refers to the office environment. The using object and the object of children's dental treatment machine are people, forming a dual interface, doctor-dental treatment interface (Human Machine Interface 1) and patient- dental treatment interface (Human Machine Interface 2 ), as shown in Fig. 1. The application of ergonomics in the design of children's dental treatment can be summarized as: to provide human static and dynamic parameters of children and doctors; to provide a friendly man-machine interface for children and doctors; to provide scientific basis for the doctor's working posture; and to provide design guidelines for the treatment machine of work space layout.

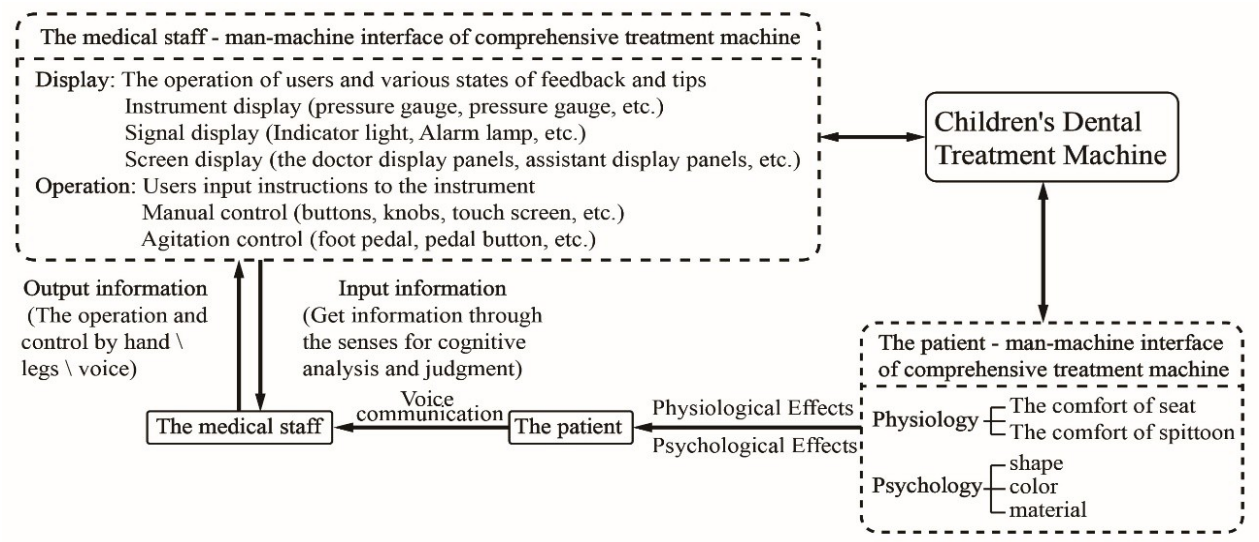

Figure 1: Dual Interface of Children's Dental Treatment Machine

\subsection{Computer Aided Ergonomics Design}


Computer Aided Ergonomics Design (CAED), refers to doing all kinds of creative design activities related to ergonomics under the support of computer and its aided design system. At present, the key technologies and methods of computer-aided ergonomics design is shown in Fig. 2:

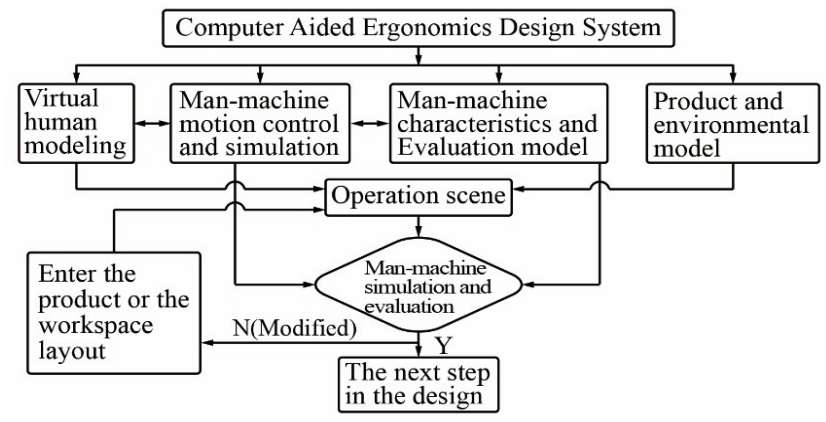

Figure 2: Computer Aided Ergonomics Design System

\subsection{Design Process Based on Ergonomic-prototype}

There are two shortcomings in traditional ergonomic design: (1) using two-dimensional human templates at the design stage and using physical prototype in the evaluation phase, instead of introducing computer-aided design, this results in the increase of the design time and cost; (2)with the introduction of computer-aided design, it does not consider ergonomics in the initial stages of the design but only in man-machine analysis and evaluation phase when the design is complete. When it fails to meet the human-machine performance, one needs to make large changes of the products.

According to the above analysis of the shortcomings of ergonomics in product design applications, this paper presents a method of product design based on the ergonomic-prototype. The design flow of ergonomic-prototype is shown in Fig. 3. Firstly, the virtual human model and virtual people working posture are determined by the related theory of ergonomic; secondly, through the man-machine analysis of the accessibility of posture, comfort, and visibility, drive the ergonomics form design of the prototype; and then based on the prototype, deepen designing the product structure, modeling, and other aspects, so as to get digital model of product design; finally, the virtual human model and product digital model are put into the operation scene for man-machine simulation evaluation and modification, until meet the man-machine performance to complete the design.

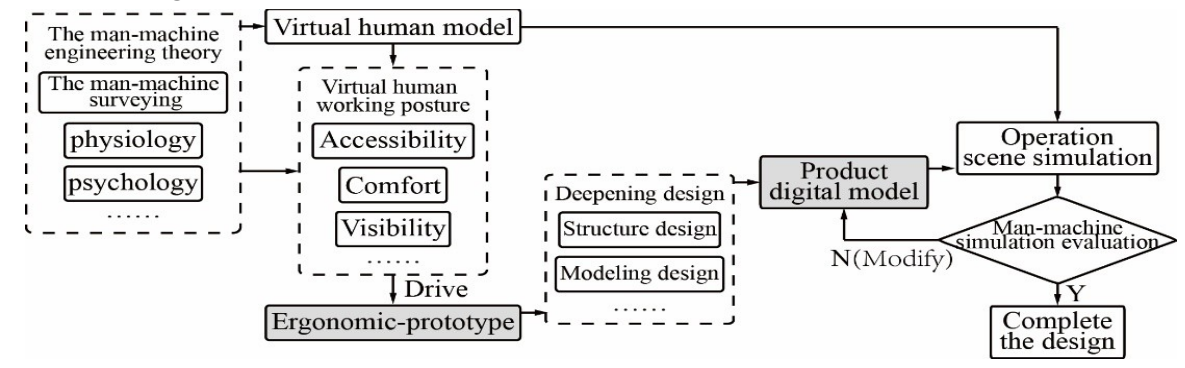

Figure 3: Design Process Based on Ergonomic-prototype

\section{Application of CAED in the Design of Children's Dental Treatment Machine}


The application of CAED technology is still at a very early stage, but because of its well solving the drawbacks of the traditional model, it will be a major trend of the development of ergonomics. The specific process is to set up a virtual human model, man-machine prototype of correct working posture, and the 3D model of product design, based on the related ergonomics theory, and then put them into the operation scene for simulation analysis of man-machine, until meeting the human-machine performance to complete the design.

\subsection{Establishment of Children's Model}

The human body model with the application of ergonomics in the design process is mainly used in the detection and analysis of human scale and data. There are no exact strict requirements on the structure of the human body and the appearance of the surface. The simplified human body is more conducive to the establishment and the late simulation and evaluation. This study refers to "Stick-man " concept to simplify the model[2].

According to the children's high oral prevalence of age and treatment compliance, the age range is $4 \sim 10$ years old. The study needs to establish a maximum and a minimum body model. The data come from GB/T 26158-2010. Fig. 4 shows the simplified model of children. The model is established by CATIA, whose surface system associates with skeletal system. Through the control of skeletal system movement, achieve the motion of virtual human, which simulates human body posture change, as shown in Fig. 5 .

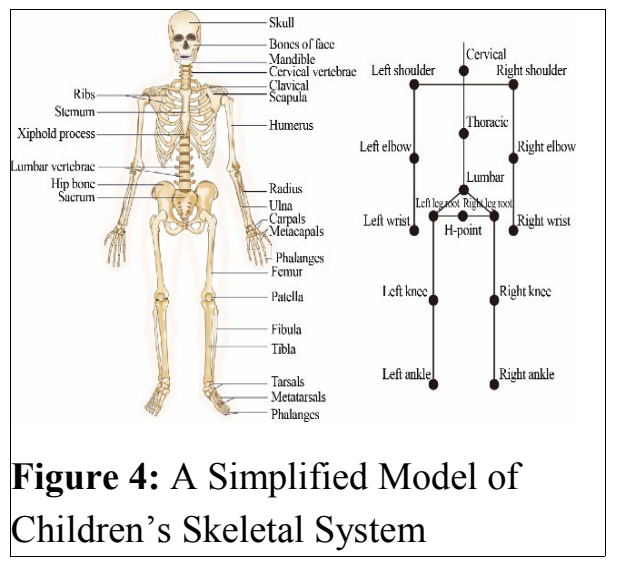

\subsection{Study on Dental Treatment Posture}

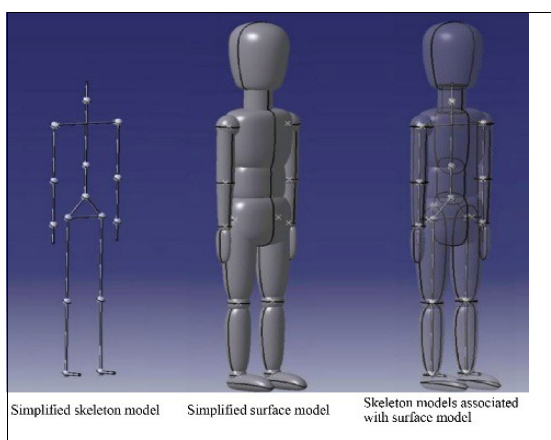

Figure 5: Surface Model Associated with Skeletal Model

Dental treatment process is a very delicate process. Dentists need to deal with all kinds of intricate treatment and prevention in view of narrow mouth, many operations only within the range of $0.1 \mathrm{~mm}$ to $0.2 \mathrm{~mm}$. Therefore the incidence of neck, shoulder, wrist syndrome and lumbago is higher than that of other occupations [3].

\subsubsection{The Theoretical Basis of Dentist's Correct Operation Posture}

PD (Proprioceptive Derivation) is proposed by Dr. Beach who was employed by Japanese HPI (Human Performance and Informatics Institute) in 1985[4]. PD theory is a strict specification for dental treatment posture, guiding dental treatment posture for hundreds of dental clinics. Proprioception refers to a sense of balance and muscle feeling within the body. It makes the behavior of people and their surrounding environment build a sense of natural balance. All natural and healthy state is considered "zero". In contrast, unnatural and unhealthy 
state is based on the extent of the "-1" to "-9". This research is based on the zero standard of various parts of the body, to draw dentist's correct posture and the standardization of patient's posture.

\subsubsection{Dentist's Correct Operation Posture}

According to the principle of PD, dentists should have a balanced position, which is shown in Fig. 6. Specific requirements are as follows: (1) the line formed by the ischial tuberosity connection, the ischial tuberosity and the fibular head parallel to the ground. Doctor's lap margin and the horizontal plane are approximately 15 degree angle; (2) the trunk axis is vertical; (3) the pupil and eye line-tragus line parallel with the ground plane. Two shoulders connection is also parallel to the ground; (4) the long axis of the upper arm is perpendicular. Elbow is contacted with the costal margin. The operating point is located in the mid sternum or the heart; (5) head leans forward slightly. Eyes look down at the work area, and distance between dentist's eyes and the patient's mouth is $36-46 \mathrm{~cm}$.

\subsubsection{The Standardization of Patient's Posture}

Since supine position is the most stable and natural posture, the patient's position should be supine or close to it. In order to cope with the PD operation posture of dentist, the standardizations of patient's posture are summarized as follows: (1) the patient's head leans forward or backward, so that the mandibular plane parallels to the horizontal plane, or the maxillary plane is perpendicular to the horizontal plane, and the angle of the front and rear of the head is between 8 and 25 degrees; (2) the head of the patient can turn around, angle between \pm 45 ; (3) changes in the patient's chair position, must take the doctor's operating point at the midpoint of the sternum or the level of the heart, allowing dentist in the correct posture to see the surgical area, which is shown right in Fig. 6.

\subsubsection{The Establishment of Dentist's Model and Simulation of Treatment Posture}

According to GB10000-88 Chinese adult human body measurements, dentist mannequins is established by CATIA. Since sitting position is the main posture, sitting body measurement is the main basis for the establishment of a dentist mannequin, as shown in Figure 6. According to the theory of PD, the PD position is the most comfortable treatment position. Simulation of PD position shown right in Fig. 7, can provide references for the operating space design of children's dental treatment machine.
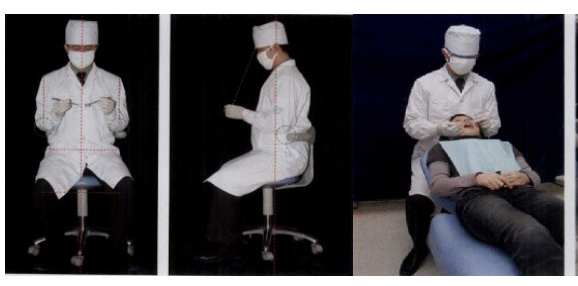

Figure 6: Doctor and Patient's Position
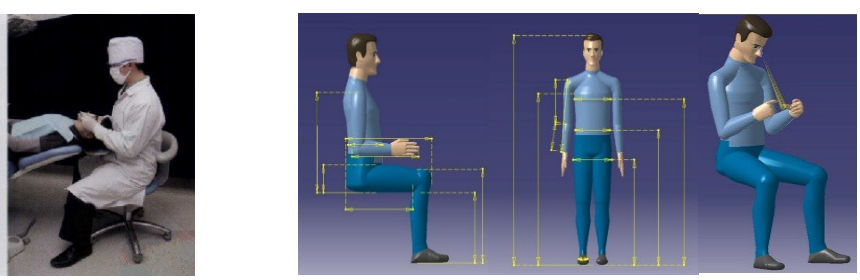

Figure 7: Dentist's Model and Posture Simulation

\subsection{The Design of Working Space for the Treatment Machine}

Working space design refers to the work in accordance with the operator's posture, scope of operation, visual range, and a series of physiological and psychological factors on machinery, equipment, tools, object for reasonable layout and arrangement, to make the operation safe and 
comfortable and improve the work efficiency [5]. The working space of medical equipment typically includes the working space of medical staff and patient's treatment space.

\subsubsection{The Requirements and Scope for the PD Treatment Operation Area}

The theory of PD has corresponding specification for doctor and assistant position. The positional relationship among doctors, assistant and patients is considered with an imaginary clock face, as shown in Fig. 8. Doctors' work area ranges from 9:30-12:30. Assistant's work area ranges from 12:30-3:30. Doctors and nurses often transfer tools from 5:00-8:00, forming the elliptic transfer zone [6].

\subsubsection{Structure Analysis of Dental Treatment Machine}

Dental Treatment Machine is equipped with an integrated platform for dental equipment. In the abstract, the commonly used equipment for the treatment of oral diseases can be integrated into comprehensive dental treatment machine. The most basic and important seven structural components are shown in Fig. 9.
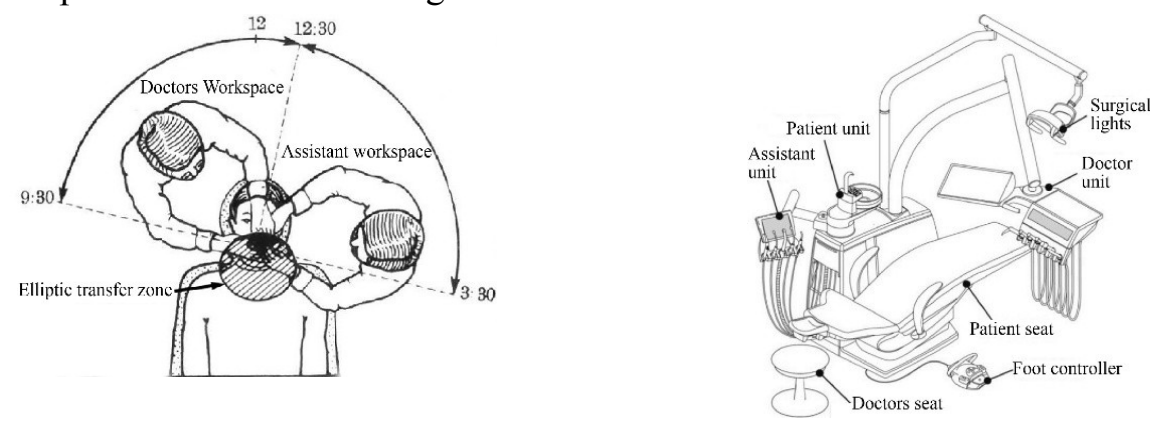

Figure 8: Doctors and Assistants Working Zoning Figure 9: Seven Structural Components

\subsubsection{Construction of the Man-machine Prototype}

Because of computer-aided ergonomics, virtual models of dental unit should be set up based on human anatomy, anthropometry, and dental medical theory of ergonomics. For instance, the dental chair backrest width consulting the children P95 percentiles of the shoulder is $374 \mathrm{~mm}$, plus $10 \mathrm{~mm}$ margin on both sides, then the width is $374+10 \times 2=394 \mathrm{~mm}$.

Through consulting relevant numerical size, each functional component is analyzed and designed, the basic dimensions of the virtual human model as shown in Table 1. And the manmachine prototype is constructed in CATIA, as shown in Fig. 10.

\begin{tabular}{|l|l|l|}
\hline \multicolumn{2}{|l|}{ Structural components } & Basic size \\
\hline \multirow{3}{*}{ Doctors seat } & Seat & Width $400 \mathrm{~mm}$; Depth $380 \mathrm{~mm}$; Angle $10^{\circ}$ \\
\cline { 2 - 3 } & Backrest & Width $320 \mathrm{~mm}$; Length $250 \mathrm{~mm}$; Angle $95^{\circ}$ \\
\hline \multirow{3}{*}{ Dental chair } & Seat & Width $319 \mathrm{~mm}$; Length $780 \mathrm{~mm}$; Thigh angle $12^{\circ}$; Leg angle $6^{\circ}$ \\
\cline { 2 - 3 } & Backrest & Width $394 \mathrm{~mm}$; Length $373 \mathrm{~mm}$ \\
\cline { 2 - 3 } & Headrest & Width $178 \mathrm{~mm}$; Length $180 \mathrm{~mm}$ \\
\hline Instrument tray & $400 \mathrm{~mm} \times 300 \mathrm{~mm}$ \\
\hline Assistant plate & $300 \mathrm{~mm} \times 225 \mathrm{~mm}$ \\
\hline Spittoon & Diameter $240 \mathrm{~mm} ;$ \\
\hline Operating lamp & $120 \mathrm{~mm} \times 80 \mathrm{~mm}$ \\
\hline Foot controller & Length $200 \mathrm{~mm}$; Width $150 \mathrm{~mm}$; Angle $12^{\circ}$ \\
\hline
\end{tabular}

Table 1: The Basic Dimensions of the Virtual Human Model 


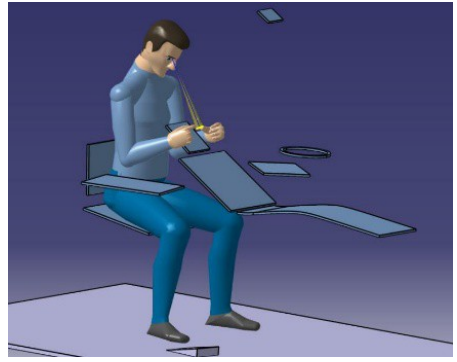

Figure 10: Man-machine Prototype in CATIA

\subsection{Detailed Design Based on Ergonomic-prototype}

Ergonomic-prototype is only a necessary limit to the size of the product, which is not limited by the specific structure and modeling. Product design in engineering practice is a complex process, which should deal with the relationship among efficiency, economy and comfort of the three. In the previous paper, the 3D ergonomic-prototype of the children's dental treatment machine has been established. Engineers and designers can perform detailed design on the basis of this prototype.

People is the center of the design and scale, including physical and psychological scale. The physiological scale refers to the basic data of the human body. Psychological scale is to meet people's needs through the design of product shape, color and materials. Children's dental treatment machine is medical equipment, and belongs to the category of children's products. Detailed design on its structure, shape, color and materials needs more attention, according to some design principles.

\subsubsection{Detailed Design of Structure Based on Ergonomic-prototype}

First, observe ergonomic-prototype in the dental chair lift, and select the appropriate institutions, and draw the schematic diagram of mechanism as shown in Fig. 11. According to the requirements of ergonomics-prototype in range of motion, establish the lifting mechanism kinematic sketch under the environment of CATIA sketches, and determine the appropriate size of the structure and position of the mounting holes through the relevant calculations, and the data are shown in Fig. 12; next, the three-dimensional model of the mechanism is established according to the size in CATIA, for observing whether the movement of the dental chair is satisfied with the movement of the dental chair, as shown in Fig. 13; finally, in accordance with the overall assembly for each part of the detailed design, dynamic simulation analysis and finite element analysis are carried out on the mechanism.

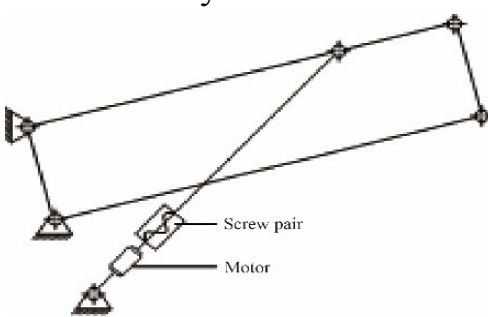

Figure11:Parallel Quadrilateral

Lifting Structure Diagram

Example

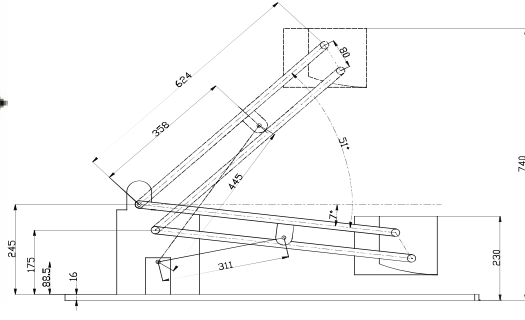

Figure12:Dimension Calculation of Figure13:Three Parallel Quadrilateral Mechanism -dimensional Model of an Mechanism 


\subsubsection{Detailed Design of Modeling Based on Ergonomic-prototype}

First, with importing the ergonomic-prototype in the software, a new canvas is selected at the appropriate view angle, for drawing the sketch as shown in Fig. 14; then the 3D model is established based on the sketch, and the result is shown in Fig. 15. The traditional digital model can only refer to the 2D image, and the 3D modeling based on the Ergonomic-prototype design can make the model designer more accurately; finally, different shape programs for visualization are shown in Fig. 16. Thus, the same individual ergonomic-prototype can carry out different ideas and modeling.

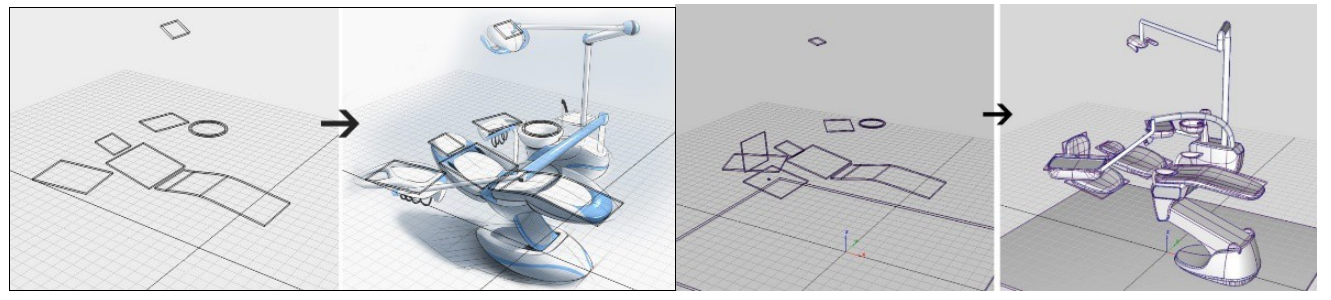

Figure 14: 3D Design Based on

Ergonomic-prototype

Figure 15: Sketch Design Based on Ergonomicprototype
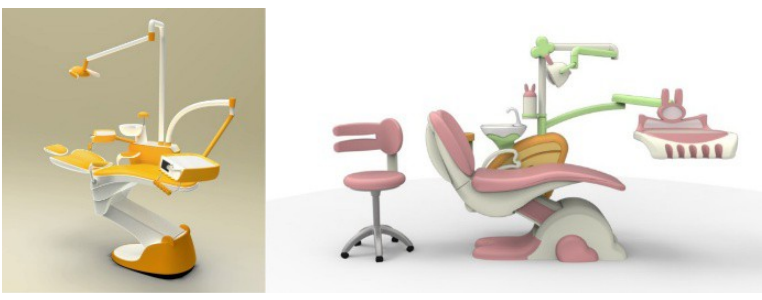

Figure 16: 3D Visual Rendering of Different Modeling Design

\subsubsection{Simulation Analysis of Children's Dental Treatment Machine}

The result of detailed design based on man-machine prototype and the prototype itself are connected in CATIA, to simulate the movement of every component for the digital virtual prototype with static and dynamic measure. On the one hand it's intuitively checked to see whether the movement of the component and the prototype are consistent on the other hand it's ready for the further man-machine simulation and evaluation. For the children's dental treatment machine, it needs simulation analysis of accessibility, comfort, visibility and other aspects of the man-machine system, to judge whether the comprehensive evaluation result meets the requirements. The simulation analysis process is shown in Fig. 17:

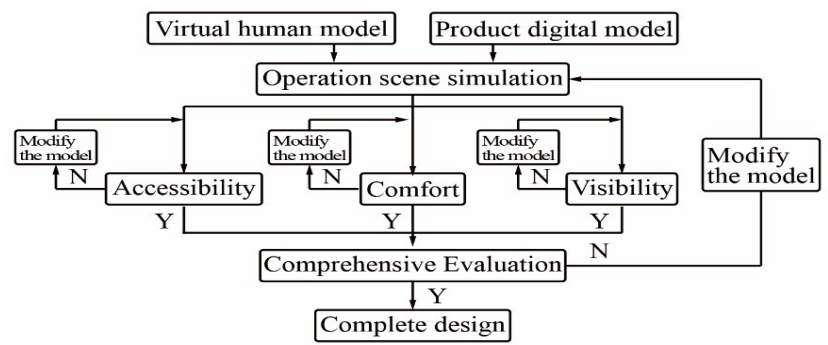

Figure 17: Simulation Analysis Process of Product Ergonomics

The design of children's dental treatment machine is driven by the treating pose of doctors and children, which means the various functional components of motion, and is to enable 
doctors to keep operating at PD treatment position. CATIA is used to analyze and evaluate the accessibility and visibility of the design. It is required to keep the best position of the doctor and the children in different body, and to verify whether the doctors' surgical area is within the optimum range of the visual field, as well as whether various functional components of the treatment machine is within reach of range, as shown in Fig. 18, Fig. 19and Fig. 20:

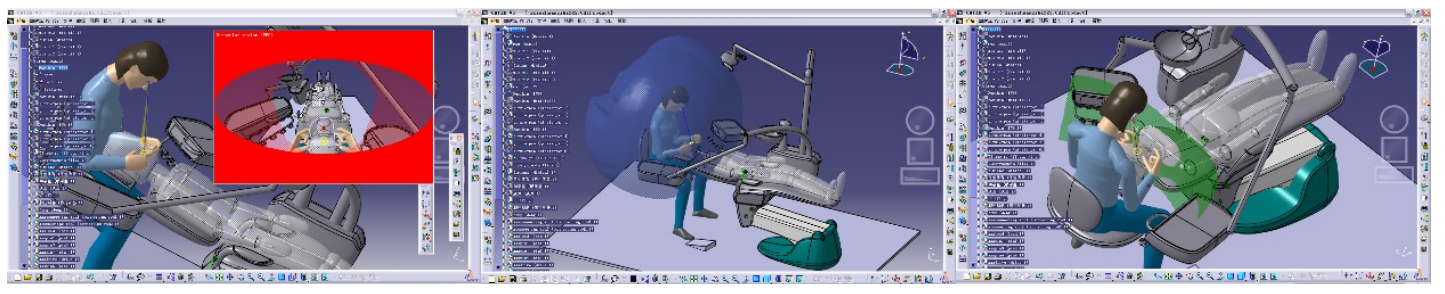

Figure 18: The Analysis of Figure 19: The Analysis of Doctor Visibility
Doctor Hands Accessibility
Figure 20: The Analysis of the Best Domain for Doctor

\section{Conclusion}

Children's Dental treatment has a complex dual man-machine interface. It is a meaningful attempt for applying computer-aided ergonomics design system. The process is to create a virtual human model and the prototypes on the basis of ergonomics theory, establish virtual prototype after detailed design, and then put them into the operation scene for simulation analysis of man-machine, until they meet the human-machine performance to complete the design. This practice can not only provide theoretical basis for the development of special children's dental treatment machine, but also provide reference on other product for the application of this system.

\section{References}

[1] Z. Li, L.X. Zhan, J. H. Zhang, J. Z. Liu, The application of human engineering in medical equipment design, China Medical Device Information, 2012, 18 (8):2 .

[2] M. Xu, Human biomechanical model simulation analysis based on ergonomics, Zhejiang University, 2006:90

[3] R.W. Marklin, K Cherney, Working postures of dentists and dental hygienists, California Dental Association, 2005, 33(2):133-136

[4] A. Gupta, M. Bhat, T. Mohammed, N. Bansal, G. Gupta International Journal of Clinical Pediatric Dentistry, 2014, 7(1):30-34

[5] L.Y Sun, Human factors engineering, Science and Technology of China Press, 2001:25-33

[6] A.Q. Lin, Dental PD basic and clinical operation, Shanghai medical university press, 1993:23-33 